\title{
Ametantrone Acetate
}

National Cancer Institute

\section{Source}

National Cancer Institute. Ametantrone Acetate. NCI Thesaurus. Code C28798.

Acetate salt of Ametantrone, a topoisomerase II inhibitor of the anthrapyrazole family that causes covalent cross-links in DNA. 